\title{
Modelling of nonlinear bridge aerodynamics and aeroelasticity: a convolution based approach
}

\author{
T. $\mathrm{Wu}^{1}$ and $\mathrm{A} . \mathrm{Kareem}^{1}$ \\ ${ }^{1}$ Nathaz Modeling Laboratory, University of Notre Dame, Notre Dame, IN 46556, USA
}

\begin{abstract}
Innovative bridge decks exhibit nonlinear behaviour in wind tunnel studies which has placed increasing importance on the nonlinear bridge aerodynamics/aeroelasticity considerations for long-span bridges. The convolution scheme concerning the first-order kernels for linear analysis is reviewed, which is followed by an introduction to higher-order kernels for nonlinear analysis. A numerical example of a longspan suspension bridge is presented that demonstrates the efficacy of the proposed scheme.
\end{abstract}

\section{Introduction}

The worldwide need to bridge wide river basins, straits and oceans has led to spans that are progressively increasing involving innovative deck sections, which exhibit aerodynamic nonlinearities. Actually, the "nonlinear" effects are usually exploited to give a possible explanation for any difference between results based on linear analysis and experiments. However, it is difficult to delineate the contributions of linear and nonlinear effects [1]. Though increasing nonlinear behaviour is observed in wind-tunnel data, current analytical methods have proven to be unable to fully represent the behaviour of these bridges characterized by aerodynamic/aeroelastic nonlinearities and turbulence effects. This limits the utility of the state-of-the-art linear analysis procedures and calls for a comprehensive nonlinear analysis framework. Thus far nonlinear effects arising from structural aspects are well understood and modelled, however, very limited models exist that attempt to simulate aerodynamic nonlinearity. This is due in part to the complexity involved and intractability of associated modelling. The nonlinear effects in bridge aerodynamics/aeroelasticity may result from the flow separation, reattachment around the deck, and the wake.

An obvious advantage of the time domain analysis is its ability to take into account the nonlinear effects readily. In time domain, the convolution of a linear kernel, e.g., the unit-step response function, is well known as the Duhamel's integral. The nonlinear analysis of bridge aerodynamics/aeroelasticity in time domain is obtained by extending the linear analysis framework utilizing a Volterra-type formalism, The identification schemes for the higher-order step or impulse response functions are introduced here. A numerical example of a suspension bridge with vertical and torsional degrees of freedom is presented. The simulation results show the significance of nonlinearity in bridge aerodynamic/aeroelastic effects.

\section{Identification of nonlinearity}

Accurate modelling of aerodynamic/aeroelastic forces is always a critical issue in ensuring the safety of long-span bridges. There are at least four nonlinear phenomena observed in bridge aerodynamics/aeroelasticity: (i). nonproportional relationship between amplitudes of input and output (ii). single-frequency input exciting multiple frequencies; (iii). hysteretic behaviour of aerodynamic/aeroelastic forces versus angles of attack and (iv). amplitude dependence of aerodynamic and aeroelastic forces.

Nonlinear behaviour (ii) is usually referred to as super-harmonic generation and harmonic distortion, which may also surface when the input constitutes of several frequency components. Fig. 1 illustrates the harmonic distortion in bridge aerodynamics using experimental data of a typical modern bridge deck. The input turbulent wind velocity has only two frequency components, $0.38 \mathrm{~Hz}$ and $1.51 \mathrm{~Hz}$. However, the torsional displacement responses of the example bridge deck not only have contributions at these frequencies as a result of linear transformation, but also $0.74 \mathrm{~Hz}$ component emerges as a super-harmonic and $1.13 \mathrm{~Hz}$ from harmonic distortion. Similar nonlinear behaviour is also observed in bridge aeroelasticity where the inputs are structural displacements and the outputs are motioninduced forces. 

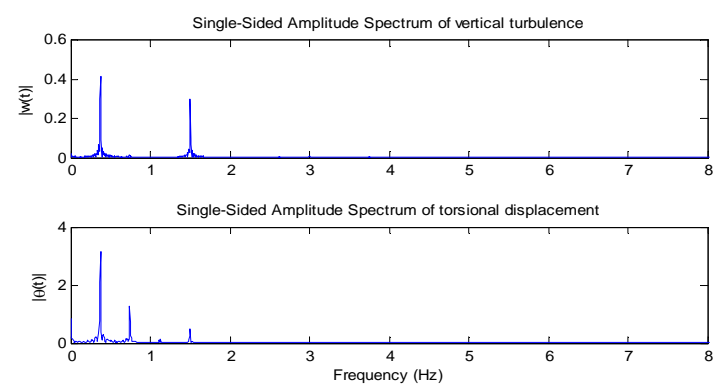

Fig. 1. Nonlinear transformation of bridge aerodynamics.

In order to further investigate the aerodynamic and aeroelastic nonlinearity, the higher-order spectra (or higher-order coherence) can be utilized, which preserve the phase information [2]. Instead of giving the contour figure as normally in the literature, Fig. 2 presents the auto-bi-coherence of two frequency components with all the frequencies within the range of interest, which clearly presents the nonlinear coupling phenomenon existing among different frequencies. Pressure fluctuations on a cable-stayed bridge measured in a wind tunnel is shown. As indicated in this figure, there is a high level of nonlinear coupling between $1 \mathrm{~Hz}$ or $1.5 \mathrm{~Hz}$ components and the frequency region between $0-4 \mathrm{~Hz}$. After this range, the nonlinear coupling among these frequency components decreases significantly. There is almost no nonlinear coupling between $1 \mathrm{~Hz}$ or $1.5 \mathrm{~Hz}$ and the frequency region higher than $8 \mathrm{~Hz}$. Overall, the level of nonlinear coupling of $1.5 \mathrm{~Hz}$ with other frequency components is lower than that of $1 \mathrm{~Hz}$.

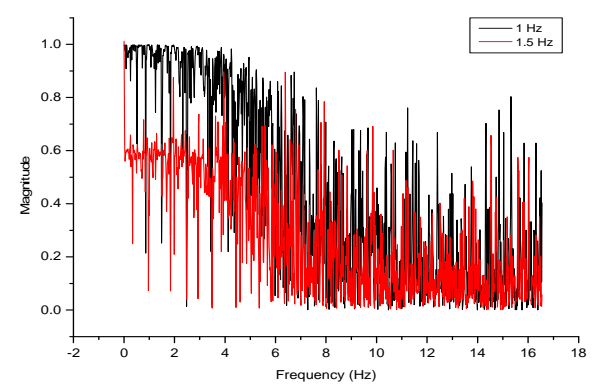

Fig. 2. Auto-bicoherence of a pressure signal.

The nonlinear behaviour (iii) observed in wind tunnel is presented in Fig. 3. Actually, hysteretic phenomenon not only indicates the nonlinearity but also the high-order memory effect, which could be well simulated by an artificial neural network [3].

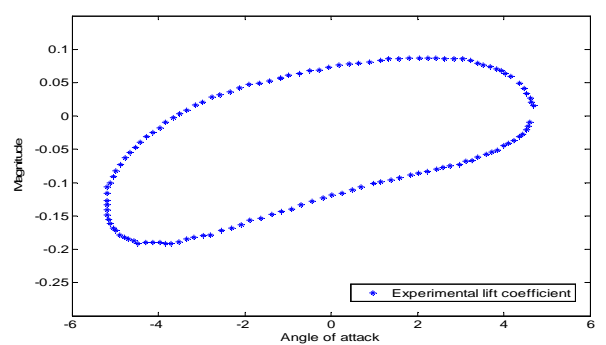

Fig. 3. Hysteretic lift coefficient (Courtesy of Prof. G. Diana).

Nonlinear behaviour (iv) is reflected in different values of the aeroelastic transfer function between the input and the output under different angles of attack, as indicated in Fig. 4. The critical wind velocity $U_{r}$ of the motion induced (self-excited) instability changes significantly under various angles of attack.

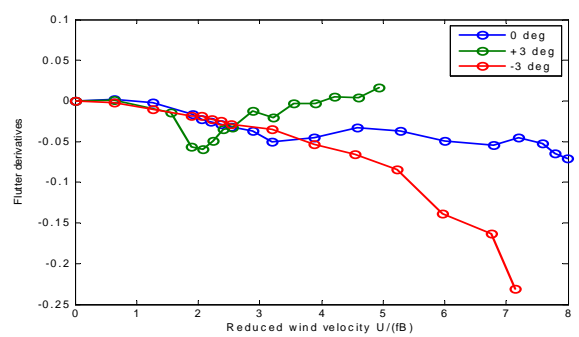

Fig. 4. Aeroelastic transfer functions (flutter derivatives).

\section{Convolution schemes}

The input information for bridge aerodynamics and aeroelasticity, when implementing convolution integrals, is an important and often misunderstood issue. Since underlying mechanisms of bridge aerodynamics and aeroelasticity are similar, therefore, without loss of the generality the discussion here is limited to the aeroelasticity for the sake of brevity. The appropriate aeroelastic inputs could be the vertical velocity $\dot{h}$, torsional displacement $\theta$ and torsional velocity $\dot{\theta}$ of the bridge deck motion in the analysis.

\subsection{Convolution of first-order kernels for linear analysis}

The linear part of the Taylor expansion of the nonlinear aeroelastic lift force $L(t)$ could be represented as

$$
L(t)=-\left[\left\{(\Delta \theta)\left(\frac{\partial L}{\partial \theta}\right)_{\theta=\theta_{0}}\right\}+\left\{(\Delta \dot{h})\left(\frac{\partial L}{\partial \dot{h}}\right)_{\dot{h}=\dot{h}_{0}}\right\}+\left\{(\Delta \dot{\theta})\left(\frac{\partial L}{\partial \dot{\theta}}\right)_{\dot{\theta}=\dot{\theta}_{0}}\right\}\right]
$$

where the steady-state part of the aeroelastic force is neglected. The nonlinear aeroelastic torsional moment $M(t)$ also has a similar expression. It is obvious that there are two time variables describing the time dependent characteristics of this system, namely, the time $t$ at which the boundary conditions (input variables) change and the time $\tau$ that indicates the duration since the change in boundary conditions.

For a time invariant system, the time dependent characteristics could be described only using one time variables $(\mathrm{t}-\tau)$. As a result, as $\Delta \theta, \Delta \dot{h}$ and $\Delta \dot{\theta}$ converge towards zero and the normalized and nondimentional aeroelastic force could be represented using a more explicit form as

$$
\begin{aligned}
L(s)=-\frac{1}{2} \rho U^{2} B[ & \frac{d C_{L}}{d \theta}\left\{\tilde{L}_{\theta}(s) \theta(0)+\int_{0}^{s} \tilde{L}_{\theta}(s-\sigma) \theta^{\prime}(\sigma) d \sigma\right\} \\
& \left.+\frac{d C_{L}}{d \frac{h^{\prime}}{B}\left\{\tilde{L}_{h^{\prime}}\right.}(s) \frac{h^{\prime}(0)}{B}+\int_{0}^{s} \tilde{L}_{\frac{h^{\prime}}{B}}(s-\sigma) \frac{h^{\prime \prime}(\sigma)}{B} d \sigma\right\} \\
& \left.+\frac{d C_{L}}{d \theta^{\prime}}\left\{\tilde{L}_{\theta^{\prime}}(s) \theta^{\prime}(0)+\int_{0}^{s} \tilde{L}_{\theta^{\prime}}(s-\sigma) \theta^{\prime \prime}(\sigma) d \sigma\right\}\right]
\end{aligned}
$$

where $\mathrm{s}$ and $\sigma$ represent non-dimensional time and $\tilde{L}_{x}(s)$ (x $=\dot{h}, \theta$ or $\dot{\theta}$ ) denotes the unit-step responses with input 
$x$. The relation between the Taylor series of a linear system and Duhamel's integral is presented by Nixon [4]. Since the unit-step response (or first-order kernel) is a function of only a single time variable, the corresponding input variable of this response is denoted as a single-step function. For the streamlined cross-section, such as the thin airfoil, the unit-step response could be derived theoretically, e.g., the Wagner function. However, for the bluff cross-section, such as the bridge deck, there is no such theoretical unit-step response as the potential flow theory and Kutta condition cannot be applied to obtain the aeroelastic force. A commonly applied approach utilizes the measured transfer function in wind tunnel (as indicated in Fig. 4) to obtained the effective unit-step responses with an indicial function or a rational function approximation scheme [5]. For example, the effective unit-step response with the input $\theta$ is presented in Fig. 5 together with the Wagner function as a comparison. The "overshooting" phenomena indicates the flow separation from the bridge deck.

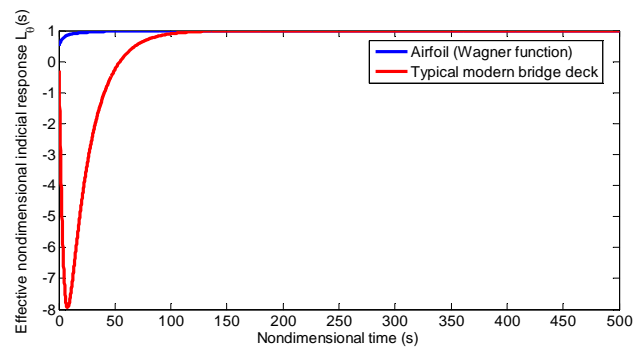

Fig. 5. Effective unit-step response.

\subsection{Convolution of higher-order kernels for nonlinear analysis}

One simple approach to analyze the nonlinear aeroelastic response is to generalize the convolution of first-order kernels. For the nonlinear consideration, the formalism of Eq. (2) is kept while the unit-step response $\tilde{L}_{x}(s)$ is a functional involving the input variables $\mathrm{x}(\kappa)[6]$. As a result, the aeroelastic force could be represented as

$$
\begin{aligned}
L(s)=-\frac{1}{2} \rho U^{2} B & {\left[\frac{d C_{L}}{d \theta}\left\{L_{\theta}\{\theta(0) ; s\}+\int_{0}^{s} \tilde{L}_{\theta}\{\theta(\kappa) ; s, \sigma\} \theta^{\prime}(\sigma) d \sigma\right\}\right.} \\
& +\frac{d C_{L}}{d \frac{h^{\prime}}{B}}\left\{L_{\frac{h^{\prime}}{B}}\left\{\frac{h^{\prime}(0)}{B} ; s\right\}+\int_{0}^{s} \tilde{L}_{h^{\prime}}\left\{\frac{h^{\prime}(\kappa)}{B} ; s, \sigma\right\} \frac{h^{\prime \prime}(\sigma)}{B} d \sigma\right\} \\
& \left.+\frac{d C_{L}}{d \theta^{\prime}}\left\{L_{\theta^{\prime}}\left\{\theta^{\prime}(0) ; s\right\}+\int_{0}^{s} \tilde{L}_{\theta^{\prime}}\left\{\theta^{\prime}(\kappa) ; s, \sigma\right\} \theta^{\prime \prime}(\sigma) d \sigma\right\}\right]
\end{aligned}
$$

where the nonlinear step-response $\tilde{L}_{x}(x(\kappa) ; s, \sigma)$ could be defined by the Fréchet derivative with respect to the input function $\mathrm{x}(\mathrm{\kappa})$. Obviously, if the system is linear $\tilde{L}_{x}(x(\kappa) ; s, \sigma)$ is equal to $\tilde{L}_{x}(s-\sigma)$. For a nonlinear system, it is convenient to involve only one input at this stage to illustrate the concept clearly without loss of the generality. For example, suppose only the torsional displacement $\theta$ is involved as the input information. If the nonlinear functional is approximated in terms of the approach similar to the generalized unit-step responses, i.e., Eq. (3), one possible form for the functional
$\tilde{L}_{x}(\theta(\kappa) ; s, \sigma)$ with respect to the input variable $\theta(\kappa)$ could be

$$
\tilde{L}_{\theta}\{\theta(\kappa) ; s, \sigma\}=\tilde{\tilde{L}}_{1 \theta}(s-\sigma)+\int_{0}^{s} \tilde{\tilde{L}}_{2 \theta}(\theta(\kappa) ; s, \sigma) \theta^{\prime}(\kappa) d \kappa
$$

By analogy, we have

$$
\tilde{\tilde{L}}_{2 \theta}(\theta(\kappa) ; s, \sigma)=\tilde{\tilde{L}}_{2 \theta}(s-\kappa, s-\sigma)+\int_{0}^{s} \tilde{\tilde{L}}_{3 \theta}(\theta(\kappa) ; s, \sigma) \theta^{\prime}(\kappa) d \kappa
$$

and

$$
\tilde{\tilde{\tilde{L}}}_{3 \theta}(\theta(\kappa) ; s, \sigma)=\tilde{\tilde{\tilde{L}}}_{3 \theta}\left(s-\kappa, s-\sigma_{1}, s-\sigma_{2}\right)+\int_{0}^{s} \tilde{\tilde{\tilde{L}}}_{3 \theta}(\theta(\kappa) ; s, \sigma) \theta^{\prime}(\kappa) d \kappa
$$

and so on. As a result, we could obtain

$$
\begin{aligned}
L(s)=-\frac{1}{2} \rho U^{2} B \frac{d C_{L}}{d \theta} & {\left[\Phi_{0 L \theta}\{\theta(0) ; s\}\right.} \\
& +\int_{0}^{s} \Phi_{\perp L \theta}(s-\sigma) \theta^{\prime}(\sigma) d \sigma \\
& \left.+\int_{0}^{s} \int_{0}^{s} \Phi_{2 L \theta}\left(s-\sigma_{1}, s-\sigma_{2}\right) \theta^{\prime}\left(\sigma_{1}\right) \theta^{\prime}\left(\sigma_{2}\right) d \sigma_{1} d \sigma_{2}+\cdots\right]
\end{aligned}
$$

Since the nonlinear step response (or higher-order kernels) is a function involving multiple time variables, the corresponding input variable of this response is denoted as a multi-step function with simultaneous input quantities. In this situation, the input and output construct the multiple convolution integral. The corresponding equivalent in terms of nonlinear impulse response could be represented as

$$
\begin{aligned}
L(s)=-\frac{1}{2} \rho U^{2} B & {\left[\int_{0}^{s} I_{L L \theta}(s-\sigma) \theta(\sigma) d \sigma\right.} \\
& \left.+\int_{0}^{s} \int_{0}^{s} I_{2 L \theta}\left(s-\sigma_{1}, s-\sigma_{2}\right) \theta\left(\sigma_{1}\right) \theta\left(\sigma_{2}\right) d \sigma_{1} d \sigma_{2}+\cdots\right]
\end{aligned}
$$

The above equation has exactly the same form as the Volterra series. The Volterra series, which is a subset of the nonlinear functional expansion shown in the formula (3) [7], is often referred to as the Taylor series with memory. Usually, the truncated form of Eq. (6) is applied to simulate a nonlinear system. As usual, the wind-bridge interaction system could be treated as "weakly" nonlinear system, it is believed that it could be simulated appropriately with the $2^{\text {nd }}$ order truncated Volterra series.

\section{Numerical example}

In lieu of solving Navier-Stokes equations to model the wind-bridge interaction system, some attempts have been made to look for tractable mathematical expressions for the fluid-structural interaction. In this research, a nonlinear analytical model is introduced to identify the linear and nonlinear impulse response functions of the simplified nonlinear wind-bridge interaction system. This new nonlinear model expressed below is not very sensitive to the initial conditions as compared to the model proposed by McKenna and Tuama [8]

$$
\left\{\begin{array}{l}
I_{1} \frac{d^{2} y_{1}}{d t^{2}}+c_{1} \frac{d y_{1}}{d t}+k\left(y_{1}+\varepsilon y_{2}\right) \\
+d y_{1} y_{2}+\beta \cos \left(y_{1}\right)(\gamma / \eta)\left[\exp \left(\eta\left(y_{2}-\kappa \sin \left(y_{1}\right)\right)\right)-\exp \left(\eta\left(y_{2}+\kappa \sin \left(y_{1}\right)\right)\right)\right]=x_{1}(t) \\
m_{2} \frac{d^{2} y_{2}}{d t^{2}}+c_{2} \frac{d y_{2}}{d t}+k\left(y_{1}+\varepsilon y_{2}\right) \\
+d y_{1} y_{2}+\alpha(\gamma / \eta)\left[\exp \left(\eta\left(y_{2}-\kappa \sin \left(y_{1}\right)\right)\right)+\exp \left(\eta\left(y_{2}+\kappa \sin \left(y_{1}\right)\right)\right)\right]=\lambda x_{2}(t)
\end{array}\right.
$$


where $\mathrm{y}_{1}$ and $\mathrm{y}_{2}$ represent the torsional and vertical displacement, respectively; $\mathrm{I}_{1}$ and $\mathrm{m}_{2}$ are moment of inertia and mass of the bridge deck, respectively; $c_{1}$ and $\mathrm{c}_{2}$ are the viscosity damping in the torsional and vertical degrees of freedom, respectively; $\mathrm{x}_{1}(\mathrm{t})$ is the aeroelastic input (specifically torsional motion of the bridge deck) while $\mathrm{x}_{2}(\mathrm{t})$ is the aerodynamic input (specifically vertical gust); $\mathrm{k}, \varepsilon, \mathrm{d}, \alpha, \beta, \gamma, \eta, \kappa$ and $\lambda$ are parameters whose value are based on the bridge structural and aerodynamic properties. In order to focus on a single input multioutput $(\mathrm{SIMO})$ case, $\mathrm{x}_{1}(\mathrm{t})$ and $\mathrm{x}_{2}(\mathrm{t})$ are not concurrently set equal to nonzero. The identification of the linear and nonlinear impulse response functions is expressed as

$\left\{\begin{array}{l}h_{1}\left(t-\tau_{1}\right)=\frac{1}{\alpha^{2}-\alpha}\left(\alpha^{2} y\left[\delta\left(t-\tau_{1}\right)\right]-y\left[\alpha \delta\left(t-\tau_{1}\right)\right]\right) \\ h_{2}\left(t-\tau_{1}, t-\tau_{2}\right)=\frac{1}{2 \beta}\left[y\left[\delta\left(t-\tau_{1}\right)+\beta \delta\left(t-\tau_{2}\right)\right]-y\left[\delta\left(t-\tau_{1}\right)\right]-y\left[\beta \delta\left(t-\tau_{2}\right)\right]\right]\end{array}\right.$

where $\alpha$ and $\beta$ are constants. This generalized identification scheme is developed based on the popularly utilized impulse response function identification scheme [9] to illustrate some specific nonlinear features of the wind-bridge interaction system (e.g., amplitude dependency).

Suppose the aeroelastic input (aeroelastic unit-impulse function) is applied to this nonlinear wind-bridge interaction system. The first-order aeroelastic kernel (linear aeroelastic impulse response), the diagonal values of second-order aeroelastic kernel (nonlinear aeroelastic impulse response), and the second-order aeroelastic kernel are shown in Fig. 6 for a specific group of parameters. The superscripts $i_{1} i_{2}$ represent the kernel of output $i_{1}$ under input $i_{2}$. The identified kernel is called as direct-kernel if $i_{1}$ is equal to $i_{2}$ and cross-kernel if $i_{1}$ is not equal to $i_{2}$. Here, only cross-kernel is presented.
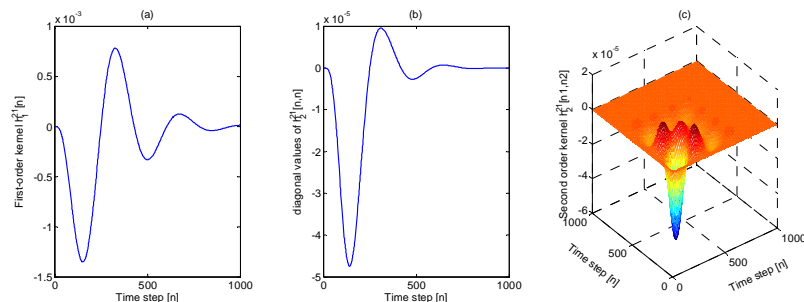

Fig. 6. Identified aeroelastic cross-kernels.

The torsional harmonic input signal (simulation of aeroelastic input) is applied to verify the identified aeroelastic impulse responses and to show the necessity of using higher-order aeroelastic impulse responses for the simulation of aeroelastic responses in this nonlinear system. Fig. 7 (a) shows the vertical response under the harmonic aeroelastic input signal using the single convolution integral (linear) only and both the single and multiple convolution integrals (linear and nonlinear).

Similar results are presented in Fig. 7 (b) for the torsional response under white-noise aerodynamic input signal (the linear and higher-order aerodynamic kernels are omitted here). As shown in both Fig. 7 (a) and (b), the inclusion of higher-order impulse response functions significantly increases the accuracy of responses for this nonlinear wind-bridge interaction system not only with respect to the amplitude but also the phase.
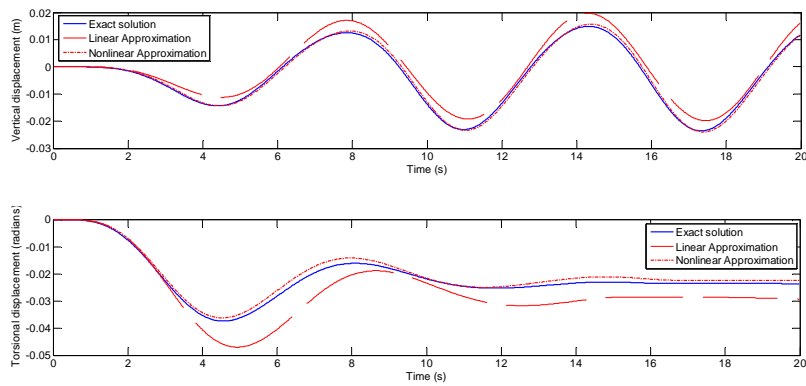

Fig. 7. (a) Vertical output $y_{2}$ and (b) Torsional output $y_{1}$.

\section{Concluding remarks}

The significance of bridge aerodynamic/aeroelastic nonlinearity is emphasized based on the observed nonlinear behaviour of long-span bridge models in wind tunnels. Based on the concept of generalized linear Duhamel's integral, the linear (single) convolution scheme is extended to the nonlinear (multiple) convolution scheme which has the form of Volterra series. Hence, a bridge aerodynamic/aeroelastic analysis framework is established in time domain which takes into account both linear and nonlinear effects based on the basic superposition concept with the elementary responses, namely, unit-step or unit-impulse response. A numerical nonlinear example of a long-span suspension bridge with two degrees of freedom is presented. The simulation results of both the aerodynamic and aeroelastic responses demonstrate the significance of nonlinearity in bridge aerodynamic/aeroelastic analysis.

The support for this project was provided by the NSF Grant \# CMMI 09-28282 which is gratefully acknowledged.

\section{References}

1. E.H. Dowell, M. Ilgamov, Studies in nonlinear aeroelasticity (Springer-Verlag, New York, 1988)

2. M.R. Hajj, W.A. Silva, J. A. 41, 1202 (2004)

3. T. Wu, A. Kareem, J. Wind. Eng. Ind. Aerodyn. 99, 378 (2011)

4. D. Nixon, Unsteady Transonic Aerodynamics (AIAA, Washington DC, 1989)

5. R.H. Scanlan, J.G. Béliveau, K.S. Budlong, J. Eng. Mech. 100, 657 (1974)

6. M. Tobak, G.T. Chapman, Nonlinear problems in flight dynamics involving aerodynamic bifurcations (NACA TM-86706, 1985)

7. P.H. Reisenthel, $34^{\text {th }}$ Aerospace Sciences Meeting and Exhibit (Reno, Nevada, 1996)

8. P.J. McKenna, C. O'Tuama, Amer. Math. Monthly, 108, 738 (2001)

9. W.J. Rugh, Nonlinear System Theory (John Hopkins University Press, Baltimore, MA, 1981) 